Pamela Johnson Rowsey $\cdot$ Bonnie L. Metzger

John Carlson · Christopher J. Gordon

\title{
Effects of chronic exercise conditioning on thermal responses to lipopolysaccharide and turpentine abscess in female rats
}

Received: 7 July 2005/ Accepted: 26 September 2005/Published online: 28 October 2005

(C) Springer-Verlag 2005

\begin{abstract}
Chronic exercise conditioning has been shown to alter basal thermoregulatory processes as well as the response to inflammatory agents. Two such agents, lipopolysaccharide (LPS) and turpentine (TPT) are inducers of fever in rats. LPS, given intraperitoneally (i.p.), involves a systemic inflammatory response whereas TPT given intramuscularly (i.m.) elicits a localized inflammation. We assessed if chronic exercise training in the rat would alter the thermoregulatory response to LPS and TPT. Core temperature $\left(T_{\mathrm{c}}\right)$ and motor activity were monitored by radiotelemetry. Female Sprague Dawley rats were divided into two groups (trained and sedentary) and housed at an ambient temperature of $22^{\circ} \mathrm{C}$. Animals voluntarily trained on running wheels for 8 weeks. In the first study, trained and sedentary female rats were injected i.p. with LPS $(50 \mu \mathrm{g} / \mathrm{kg})$ or an equal volume of $0.9 \%$ normal saline. In another study, trained and sedentary female rats were injected i.m. with TPT $(10 \mu \mathrm{l}) / \mathrm{rat}$ or an equal volume of $0.9 \%$ normal saline. The time course of the LPS fever was very short compared to TPT. TPT injected animals displayed a smaller but more prolonged fever compared to LPS; however, training accentuated the febrile response to LPS $\left(\Delta T_{\mathrm{c}}=0.6^{\circ} \mathrm{C}\right.$ in sedentary and $1.2^{\circ} \mathrm{C}$ in trained). Training had a slight suppression on TPT-induced fever during the daytime but had no effect on motor activity or nighttime $T_{\mathrm{c}}$. In
\end{abstract}

P. J. Rowsey $(\varangle) \cdot$ J. Carlson

School of Nursing, The University of North Carolina

at Chapel Hill, Chapel Hill, NC 27599, USA

E-mail: pjrowsey@unc.edu

Tel: + 1-919-5410417

Fax: +1-919-5414416

C. J. Gordon

Neurotoxicology Division,

National Health and Environmental Effects Research Laboratory, U.S. Environmental Protection Agency, Research Triangle Park, NC 27711, USA

B. L. Metzger

School of Nursing, Division I- Acute and Long-term Care Nursing, The University of Michigan-Ann Arbor, Ann Arbor, MI 48109, USA contrast, exercise training led to a marked increase in the pyrogenic effects of LPS. We conclude that the effect of exercise training and source of infection (i.e., systemic versus localized in muscle) on fever is directly linked to type of pyrogenic agent.

\section{Introduction}

Fever is a common manifestation of injury, tumor growth, infection, and inflammation. Lipopolysaccharide (LPS), a classic laboratory pyrogen extracted from the cell walls of gram-negative bacteria, causes a sudden, rapid fever when injected intraperitoneally (i.p.) or intravenously (i.v.). Most experimental studies have used LPS as the pyrogenic stimulus and have reported rapid and dramatic increases in circulating IL-6 (Klir et al. 1994), with transient increases in IL-1 (Cannon et al. 1990) and TNF (Michie et al. 1988). In response to a relatively low dose of LPS, rats and mice develop $\sim 1$ $2{ }^{\circ} \mathrm{C}$ fever that can last 4-8 hours following injection.

In contrast, TPT injected intramuscularly (i.m.) causes a localized inflammation and elicits a gradual, slow developing fever (Leon 2002). The slow acting, localized inflammatory agent TPT is also a pyrogenic stimulus. TPT acts directly on many tissues, including the brain, and unlike LPS, only very small doses are required to obtain the same physiological responses, i.e., increases in body temperature, hypothalamic-pituitary-adrenal activation and an increase in acute phase proteins (Cartmell et al. 2000; Luheshi et al. 1997). An increase in plasma IL-6 has been associated with the febrile response of TPT (Cartmell et al. 2000). However, blockers to TNF- $\alpha$ (Cooper et al. 1994) and IL-1 (Luheshi et al. 1996) significantly inhibit the fever and increased plasma IL-6 concentration elicited by TPT injection.

Chronic exercise training has a number of beneficial effects on the immune system and host resistance that could modulate the physiological response to fever 
(Cannon and Kluger 1983; Ostrowski et al. 1998, 1999; Pedersen 2000; Pedersen and Toft 2000; Sugimoto et al., 2000). It has been shown that exercise in rats (Rowsey et al. 1993a, b; 2001, 2003; Rowsey and Kluger 1994; Satinoff et al. 1991; Sugimoto et al. 2000) and hamsters (Conn et al. 1990) results in a relatively large rise in core body temperature during the nighttime, when rodents are active or exercising, and small increases in the daytime, when rodents are inactive (Rowsey et al. 1993a, b, 2001, 2003; Rowsey and Kluger 1994). The nighttime rise in core temperature $\left(T_{\mathrm{c}}\right)$ is attributed to the increase in heat production from running. It remains to be seen, however, whether the exercise-induced elevation in daytime temperature involves mechanisms characteristic of an infectious fever or fever associated with an inflammatory response. None of the studies to date have investigated the effects of long-term exercise conditioning (physical training) on the mechanisms of rapid and slow developing fevers.

The study reported here was designed to see if exercise training would alter the physiological expression of the sudden rapid and slow developing fevers induced by LPS and TPT, respectively. Since long-term exercise conditioning has been shown to cause nonspecific immune changes, e.g. changes in temperature and an increase in cytokine production, similar to those induced by LPS and TPT, we hypothesized that exercise training would alter the effects of the nonspecific immune response induced by LPS, a sudden rapid fever, and TPT, a slow developing fever.

\section{Materials and methods}

Sixty-four young adult female Sprague Dawley rats were used in the two studies (LPS study, $n=32$ and TPT study, $n=32$ ). Animals were obtained from Charles River Laboratories (Raleigh, NC, USA) at 45 days of age weighing between 90 and $110 \mathrm{~g}$. The rats were housed individually in acrylic cages lined with wood shavings and maintained at an ambient temperature (Ta) of $22^{\circ} \mathrm{C}$ and $12: 12 \mathrm{light} /$ dark photoperiod (lights on at $0600 \mathrm{~h}$ ). Animals were allowed at least 1 week to acclimate to the animal facility before radio transmitters were implanted. Rats were fed standard rodent chow and given water ad libitum.

Each animal was surgically implanted with a radio transmitter (Data Sciences International, St. Paul, MN, USA) to provide telemetric monitoring of core temperature and motor activity as described previously (Gordon 1994). Briefly, animals were anesthetized with sodium pentobarbital $(50 \mathrm{mg} / \mathrm{kg}$, i.p.), and a small incision was then made in the ventromedial section of the abdomen to allow for insertion of the transmitter (Data Sciences, model TA10TA-F40) into the peritoneal cavity. As a prophylactic measure, each animal received 30,000 units, i.m. of penicillin immediately after surgery. The rats were allowed at least 1 week of recovery prior to being placed on running wheels. Rats were then divided by weight into exercise (provided running wheels) and sedentary (no wheels) groups.

The output of the transmitter was monitored at 10minute intervals by a receiver board placed under each rat's cage. Motor activity was measured from the change in position of the transmitter in relation to the antennae located in the receiver board. Baseline core temperature and motor activity were obtained at least $48 \mathrm{~h}$ before experimentation, while animals remained in their home cage undisturbed in the animal facility.

\section{Exercise protocol}

Animals were housed in a specifically designed cage with a running wheel (Nalge designed for Minimitter Company, Sun River, OR, USA) that allowed physiological telemetric monitoring. Dimensions of fully assembled cages with the wheel were (length $\times$ width $\times$ height) $50 \times 26.8 \times 36.4 \mathrm{~cm}^{3}$.

A "training effect" was defined as a consistent daytime elevated body temperature of exercised animals above sedentary controls. Previous studies (Rowsey et al. 1993a, b, 2001, 2003; Rowsey and Kluger 1994) have shown that female Sprague Dawley rats voluntarily run with sufficient duration and intensity to result in an elevation of their day and nighttime core temperature. After placement in the cage with running wheels, the exercise animals showed changes in nighttime core temperature almost immediately. An elevation in daytime core temperature became apparent after approximately 2 weeks of running on wheels. Wheel revolutions were recorded (revolutions per minute) each time an electronic counter was engaged and an infrared beam was broken.

\section{Protocol}

\section{Turpentine study}

After 8 weeks of housing with running wheels or no running wheels, the exercise and sedentary rats were randomly assigned to receive either $0.9 \%$ sterile saline or $10 \mu \mathrm{l}$, i.m. at $1000 \mathrm{~h}$. Half of the exercise group and half of the sedentary group were injected with TPT. The dose of TPT used in this study has previously been shown to cause a significant elevation in core temperature (Luheshi et al. 1997). Control animals received an equal volume of $0.9 \%$ sterile saline, im. Each of the four groups was weighed immediately before injection. After the initial injection of $0.9 \%$ sterile saline or TPT, the exercise animals were allowed access to their running wheels, and the sedentary animals were placed back in their cages, with no wheels. Core temperature and motor activity were monitored in both exercise and sedentary animals for 2 days. Wheel revolutions (exercise animals only) were also monitored. 


\section{LPS study}

After 8 weeks of housing with running wheels or no running wheels, the exercise and sedentary rats were randomly assigned to receive either $0.9 \%$ sterile saline or $50 \mu \mathrm{g} / \mathrm{kg}$ LPS, i.p. at $1000 \mathrm{~h}$. Half of the exercise group and half of the sedentary group was injected with LPS, extracted from Escherichia coli (Sigma, lot \#63H4011), and dissolved in filtered $0.9 \%$ sterile saline. The dose of LPS was chosen because it has previously been shown to induce a fever response that lasted for approximately 3$4 \mathrm{~h}$ (Gordon and Rowsey 1998). Each of the four groups was weighed prior to injection. After injection, the exercise animals were allowed access to their running wheels, and the sedentary animals were placed back in their cages, with no wheels. Core temperature and motor activity were monitored in both exercise and sedentary animals for 3 days. Wheel revolutions (exercise animals only) were also monitored.

\section{Data analysis}

Multivariate repeated measures analysis of variance (ANOVA), using daytime temperatures at pre-injection and 2 days post-injection, was applied to each set of measurements to determine whether long-term exercise conditioning had an effect on the response to fever from LPS, a short-acting pyrogen, and TPT, a pyrogen that produces a gradual, slow fever.

Both daytime and nighttime temperatures were calculated for each animal as the mean temperature. For each 24-hour day, daytime temperature was represented by the mean of the 10-minute observations taken from 1000 to $1350 \mathrm{~h}$, and nighttime temperature was represented by the mean of the 10-minute observations taken from 1900 to $2250 \mathrm{~h}$. The Bonferroni adjustment was used to control the Type-1 family-wise error rate at 0.05 .

The change in core temperature is based on mean temperature $24 \mathrm{~h}$ prior to dosing and 2 days after administration of saline and/or LPS or TPT. The change in motor activity was calculated from the relative change in motor activity $24 \mathrm{~h}$ before and 2 days after dosing of saline and LPS and/or TPT. The change in core temperature and motor activity over this period was calculated and analyzed for statistical significance using a two-way ANOVA followed by a Tukey's $t$-test.

\section{Results}

Effects of exercise training on core temperature

With no access to running wheels, core temperature and motor activity exhibited a typical circadian pattern for all animals (Table 1). Exercise animals ran primarily at night (approximately $50 \%$ of their nighttime) and averaged a total distance of $7.6 \mathrm{~km}$. There was little or no activity during the daytime, except when rats were
Table 1 Baseline (pre-running) mean \pm SE core temperature and motor activity

\begin{tabular}{lll}
\hline Group A: LPS & Temperature & Motor activity \\
\hline Exercise & & \\
Day & & \\
Night & $37.24 \pm 0.03^{\circ} \mathrm{C}$ & $2.93 \pm 0.20$ counts \\
Sedentary & $37.96 \pm 0.02^{\circ} \mathrm{C}$ & $7.21 \pm 0.21$ counts \\
Day & & \\
Night & $37.20 \pm 0.03^{\circ} \mathrm{C}$ & $1.76 \pm 0.22$ counts \\
& $37.97 \pm 0.02^{\circ} \mathrm{C}$ & $4.12 \pm 0.21$ counts \\
Group B: TPT & Temperature & Motor activity \\
\hline Exercise & & \\
Day & & \\
Night & $37.12 \pm 0.02^{\circ} \mathrm{C}$ & $2.43 \pm 0.17$ counts \\
Sedentary & $38.07 \pm 0.01^{\circ} \mathrm{C}$ & $8.03 \pm 0.17$ counts \\
Day & & \\
Night & $37.19 \pm 0.02^{\circ} \mathrm{C}$ & $2.03 \pm 0.14$ counts \\
\hline
\end{tabular}

disturbed such as for cage change or handling when injected. After 8 weeks of access to running wheels, there was a significant elevation in daytime and nighttime core temperature of the exercise rats (Fig. 1a, b). On the average, core temperature of the exercise group was $0.3^{\circ} \mathrm{C}$ higher than that of the sedentary group during the daytime when there was little or no running activity. During the nighttime, the core temperature of the exercise group was $0.5^{\circ} \mathrm{C}$ higher than that of the sedentary group (Fig. 1a, b).

\section{Effects of exercise training on turpentine fever}

Injection of TPT or saline induced a stress-induced rise in core temperature and motor activity in both exercise and sedentary groups, which lasted several hours (Fig. 2). Once core temperature subsided from the stress of handling, TPT had no effect on daytime core temperature during the first nine hours post-injection (first day) in sedentary and trained groups. At night, (13-24 h post-injection), the core temperatures of exercise animals that were given saline and TPT were the same. During the same time period (13-24 h post-injection), core temperature of sedentary animals receiving TPT remained slightly higher than that of the sedentary animals receiving saline; however, there was no significant nighttime effect of TPT. Motor activity counts of salineinjected animals were higher the first night after injection compared to that of the exercise animals (Fig. 3).

Twenty-four hours after injection $(25-36 \mathrm{~h}$ postinjection), the exercise rats that were given TPT showed a higher daytime core temperature than exercise rats that received saline. Sedentary animals that were given TPT also showed a higher daytime core temperature than sedentary animals that received saline. However, TPT had no effect on nighttime core temperature $(37-48 \mathrm{~h}$ post-injection) of exercise rats. Throughout the second night post-injection, core temperature of sedentary animals injected with TPT remained higher than that of the sedentary animals injected with saline (Fig. 2). 

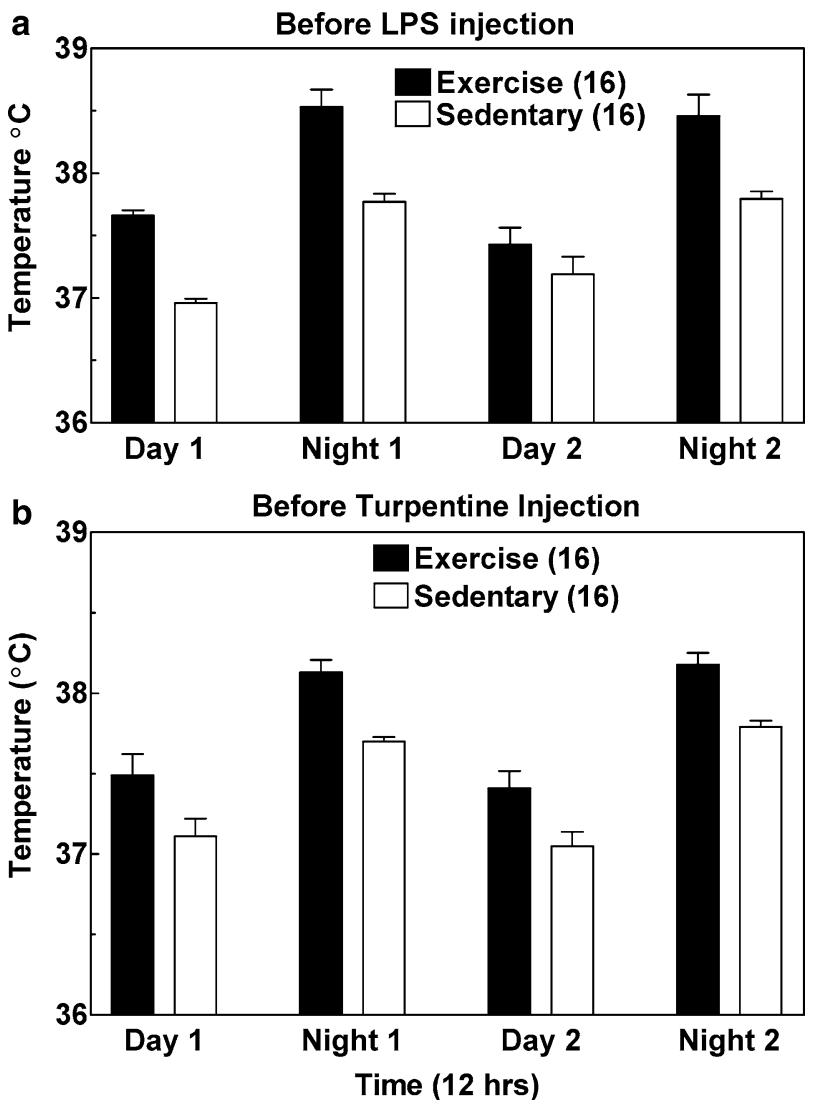

Fig. 1 Time course of daytime (Day) and nighttime (Night) core temperature after 8 weeks of rats running voluntarily on exercise wheels (Exercise) and rats with no running wheels (Sedentary), prior to LPS or TPT injection. Day represents $12 \mathrm{~h}$ lights on and night represents $12 \mathrm{~h}$ lights off

Effects of exercise training on LPS fever

Administering the saline vehicle and LPS to the sedentary and exercise animals resulted in a transient elevation in core temperature (Fig. 4) and motor activity (Fig. 5) that was attributed to the stress of handling and injection. Exercise training led to a marked increase in the pyrogenic effects of LPS. That is, both sedentary and exercise animals underwent an elevation in daytime core temperature following LPS, but the febrile response was greater in the exercise group. LPS increased the core temperature of the exercise rats about $1.3^{\circ} \mathrm{C}$ at $4 \mathrm{~h}$ postinjection while the sedentary rats' peak core temperature increase was about $0.7^{\circ} \mathrm{C}$ at $5 \mathrm{~h}$ post-injection. Core temperature of the LPS-treated animals remained elevated throughout the remaining daytime period. Core temperature of the trained rats receiving saline remained elevated about half of the nighttime period, which could be attributed to their nighttime running (data not shown).

There was little or no change in motor activity of the exercise and sedentary groups in spite of a transient increase in core temperature following injection (Fig. 5). During the daytime, motor activity was almost identical in both exercise and sedentary animals. At night, motor
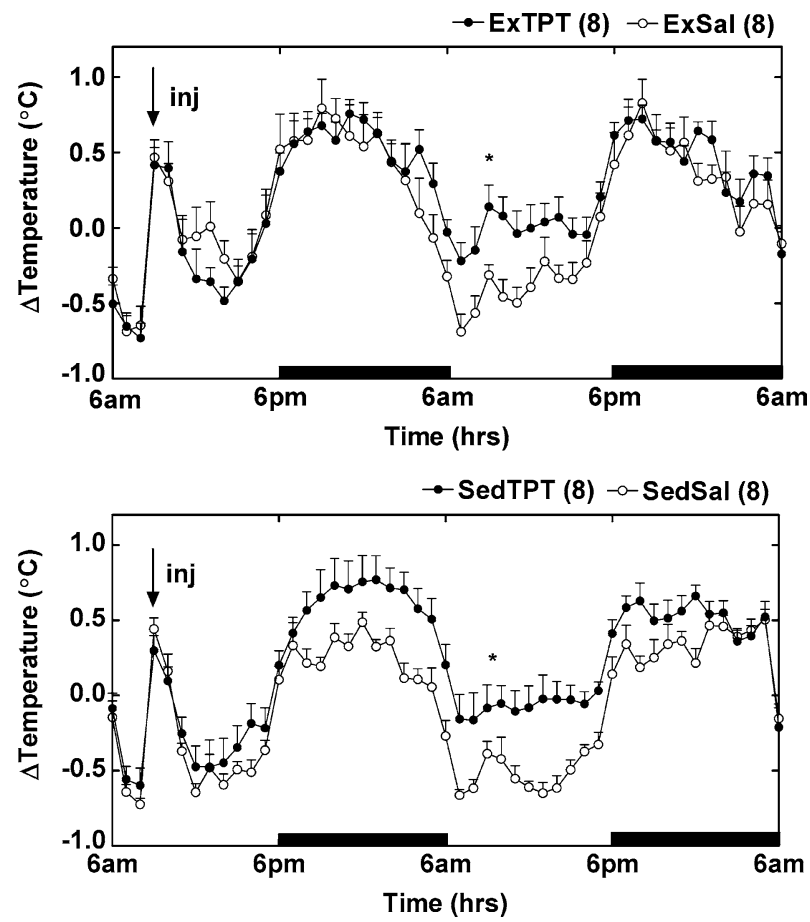

Fig. 2 Time course of core temperature of female Sprague Dawley rats injected i.m. with TPT, $10 \mu \mathrm{l} /$ rat or physiological saline. Arrow indicates the time animals were dosed. Dark horizontal bar represents the dark phase of the photoperiod. Asterisk indicates significant difference between exercise and sedentary groups. Injection day, $p=0.32$ treatment-interaction, $p=0.78$; day 1 postinjection, $p<0.01$, treatment-interaction, $p=0.007$; day 2 postinjection, $p=0.62$, treatment-interaction, $p=0.55$; day 3 postinjection, $p=0.41$, treatment-interaction, $p=0.25$. Overall, nighttime temperatures did not differ over the entire study period, $p=0.68$. Significant increase in nighttime temperature of sedentary animals, $p=0.02$

activity of the exercise animals was slightly higher than in the sedentary animals.

\section{Discussion}

This study used exercise training in an animal model to assess its putative impact on the thermoregulatory responses to two inflammatory agents. Regular voluntary running altered the fever response to two different pyrogenic agents: LPS (infectious) and TPT (local inflammatory). LPS generally elicits a large, but brief fever and TPT, elicits a smaller but long-lasting fever response.

Rats exposed to exogenous pyrogens such as LPS will maintain $\sim 1^{\circ} \mathrm{C}$ fever that lasts $4-8 \mathrm{~h}$ following injection. LPS induces the production of many proinflammatory cytokines that trigger an elevation in thermoregulatory set point (fever) by causing the synthesis and release of prostaglandin in the anterior hypothalamus (Klir et al. 1994; Michie et al. 1988; Leon 2002). In this study, following exposure to LPS, both sedentary and exercise rats responded with a similar transient increase in core temperature as a result of handling from injection. The 

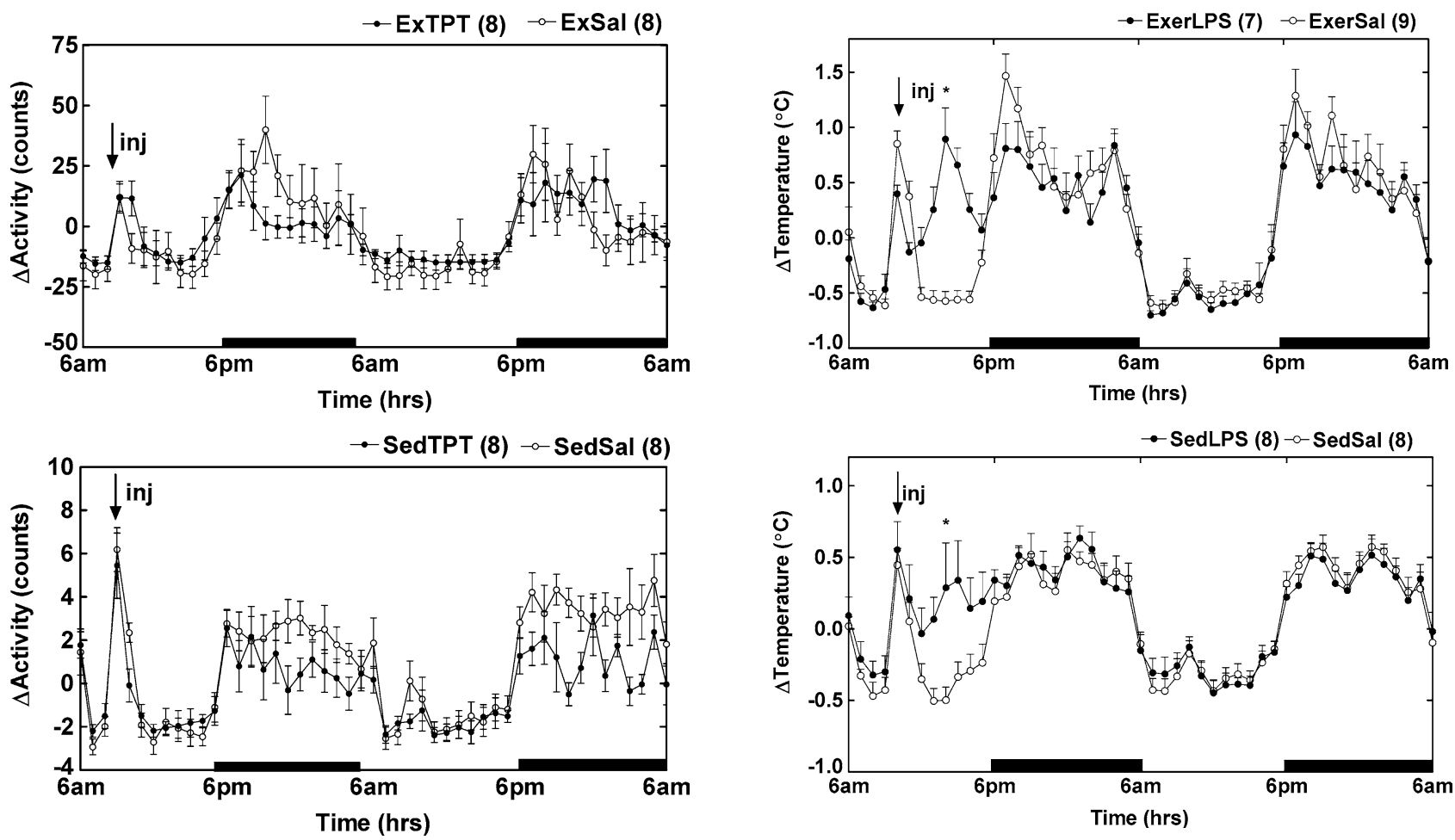

Fig. 3 Time course of motor activity of female Sprague Dawley rats injected i.m. with TPT, $10 \mu \mathrm{l} /$ rat or physiological saline. Abbreviations are the same as in Fig. 2. Injection day, $p=0.57$ treatment-interaction, $p=0.61$; day 1 post-injection, $p<0.08$, treatment-interaction, $p=0.37$; day 2 post-injection, $p=0.01$, treatment-interaction, $p=0.45$

rise in temperature (fever response) post-LPS injection between sedentary and trained animals was greater in the trained animals. Conn et al. (1990) also found $T_{\mathrm{c}}$ to be higher $\left(\sim 1.1^{\circ} \mathrm{C}\right)$ in trained hamsters administered LPS $(50 \mu \mathrm{g} / \mathrm{kg})$ compared to their sedentary counterparts.

In contrast to LPS, TPT induces a localized nonspecific inflammatory response consisting of fever, anorexia, cachexia, and acute phase protein production (Klir et al. 1994). When exposed to a relatively small dose of TPT, both exercise and sedentary animals in this study responded with a transient increase in core temperature as a result of handling. The next day, the fever response was evident in both exercise and sedentary animals challenged with TPT that lasted throughout the daytime period, suggesting that the delayed nonspecific inflammatory response induced by a local inflammatory agent is not enhanced by exercise conditioning as is seen in the acute nonspecific immune response induced by a pyrogenic infectious agent (LPS).

Just as in exercise, during infection (LPS) and localized inflammatory (TPT) exposure, there is an elevated core temperature, during the animal's nonactive period (daytime) that persists for several hours to days after exposure. It is also important to note that the stage of estrous for each animal was not determined because the animal would become very stressed during that procedure and consequently, temperatures would increase.

Fig. 4 Time course of change in core temperature of female Sprague Dawley rats injected i.p. with LPS, $50 \mu \mathrm{g} / \mathrm{kg}$ or physiological saline. Arrow indicates the time animals were dosed. Asterisk indicates significant difference between exercise and sedentary groups. Injection day, $p<0.005$ treatment-interaction, $p=0.001$; day 1 post-injection, $p=0.048$, treatment-interaction, $p=0.099$; day 2 post-injection, $p=0.500$, treatment-interaction, $p=0.535$; day 3 post-injection, $p=0.262$, treatment-interaction, $p=0.260$. Nighttime temperatures did not differ over the entire study period (4 days), $p=0.10$

Checking the estrous of each animal would ultimately interfere with the resting daytime temperatures of these animals. In addition, the increase in daytime temperature is most likely not a result of the estrous cycle. The stage of estrous appears to have little effect on the accuracy of the thermoregulatory system during the day (Yang et al. 1996).

In this study, daytime motor activity was almost identical in sedentary and exercise animals. Thus, the elevated daytime core temperature after exercise training cannot be attributed to differences in activity, but to a regulated response that could be cytokine mediated. Exercise stimulates a number of the body's defensive responses, which are similar to the responses that occur during infection and inflammation. For example, in all three events (exercise, infection, and inflammation), cytokines are released into the circulation and mononuclear lymphocytes are activated (Luheshi et al. 1997; Ostrowski et al. 1998, 1999; Pedersen 2000; Pedersen and Toft 2000; Nara et al. 1999; Sprenger et al. 1992). Thus, endogenous cytokines play a role in the induction of the acute phase response under various conditions unrelated to infection or inflammation. It can be hypothesized that the exercise-induced elevation in core temperature and release of endogenous cytokines may 


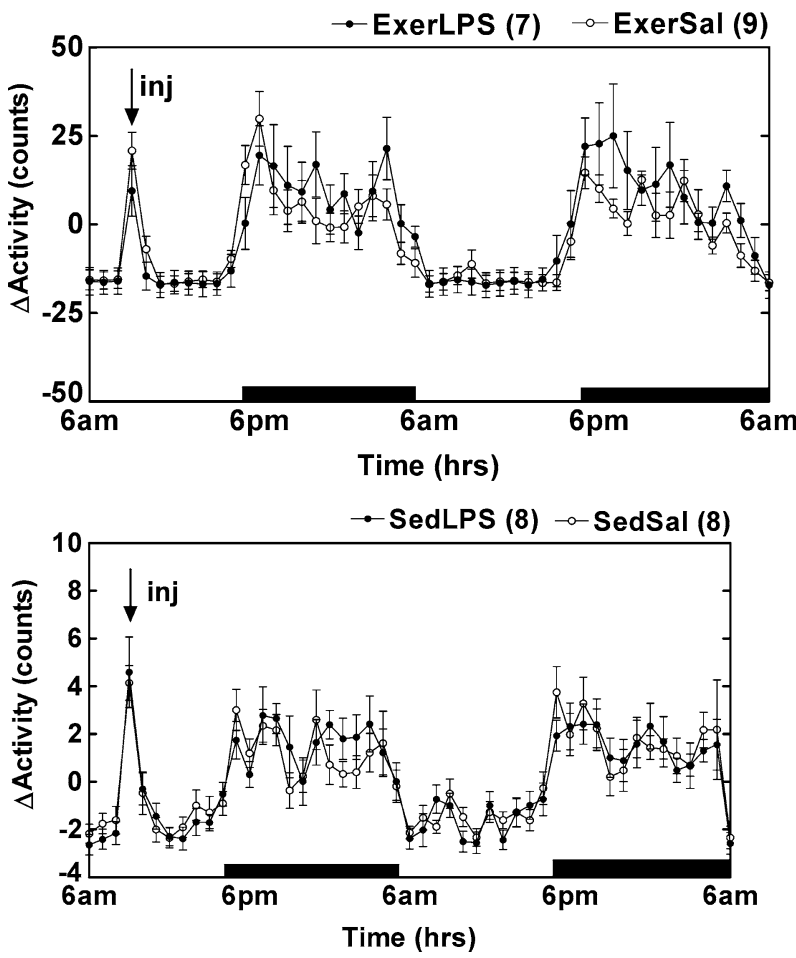

Fig. 5 Time course of motor activity of female Sprague Dawley rats injected i.p. with LPS, $50 \mu \mathrm{g} / \mathrm{kg}$ or physiological saline. Abbreviations are the same as in Fig. 2. Injection day, $p<0.088$, treatment-interaction, $p=0.132$; day 1 post-injection, $p=0.118$, treatment-interaction, $p=0.807$; day 2 post-injection, $p=0.209$, treatment-interaction, $p=0.269$; day 3 post-injection, $p=0.431$, treatment-interaction, $p=0.686$

represent a nonspecific immune response designed to defend the host against infection, inflammation or other environmental assaults by modulating the activity of endogenous cytokines.

Exercise induces numerous changes in various components of the immune system. This study lends some support to the hypothesis that long-term exercise conditioning enhances one aspect of nonspecific host defense, the acute phase response. The greater change in temperature in response to injection of LPS in exercising rats may be attributed to increase production of the cytokine, interleukin-6 (IL-6). Working skeletal muscles produce large quantities of IL-6 and may be linked to some of the beneficial effects to health when one is physically active (Ostrowski et al. 1998).

We concluded that as a result of the long-term exercise conditioning, this change in thermoregulatory setpoint allowed exercise animals to react with a greater acute phase response, i.e., change in temperature, when injected with either LPS or TPT. Since there was no difference in activity levels, we also speculate that exercise enhanced the acute phase response by promoting increased release of pyrogenic cytokines such as IL-1 $\beta$ or IL-6, which are known to induce fever and acute phase responses.

Although animal models and human studies suggest a link between exercise-induced immune changes, the clinical significance of these changes has yet to be determined. The influence of a well-prescribed amount of physical activity on immune function has important implications for individual health, as well as disease prevention and management. The survival and rigor of the population will depend on cost-effective strategies for health promotion, both in health and disease.

The effects of exercise and exercise-based immunoregulation may represent one of the frontiers for healthcare. For example, individuals are constantly exposed to nonnutrient chemicals in the environment, many of which can be toxic. One such agent is chlorpyrifos. A commonly used organophosphate insecticide, chlorpyrifos affects thermoregulation, causing an acute period of hypothermia followed by a delayed fever that lasts for $72 \mathrm{~h}$. In previous studies, exercise training attenuated the hypothermic and febrile effects of repeated (Rowsey et al. 2003) and single chlorpyrifos exposure (Rowsey et al. 2001). On the other hand, exercise training had little effect on TPT fever. Exercise training may not have as much of an effect on localized inflammation as in a systemic response such as LPS or chlorpyrifos. Data from these studies and the current study have led us to believe that while the rise in daytime temperature of rats that voluntarily exercise at night is a regulated response resulting in nonspecific immune changes (i.e., increase in thermoregulatory set-point), these nonspecific immune changes altered both the hypothermia associated with chlorpyrifos exposure and the fever responses associated with chlorpyrifos, LPS and TPT exposure. However, it is possible that the differential response of the exercised rats resulted not only from different pyrogenic agents but also from different kinetics of the agents due to the route of administration (i.p. versus i.m.). It will be important in future studies to address how route of dosing would effect exercise and fever.

Acknowledgments This project was supported by RO1 grant from the National Institute of Nursing Research, grant \#5-RO1NR04920. This paper has been reviewed by the National Health and Environmental Effects Research Laboratory, U. S. Environmental Protection Agency and approved for publication. Mention of trade names or commercial products does not constitute endorsement or recommendation for use.

\section{References}

Cannon JG, Kluger MJ (1983) Endogenous pyrogen activity in human plasma after exercise. Science 220:617-619

Cannon JG, Tompkins RG, Gelfland JA, Michie HR, Stanford GG, van der Meer JW, Endres S, Lonnemann G, Corsetti J, Chernow B (1990) Circulating interleukin-1 and tumor necrosis factor in septic shock and experimental endotoxin fever. J Infect Dis 161:79-84

Cartmell T, Poole S, Turnbull AV, Rothwell NJ, Luheshi GN (2000) Circulating interleukin-6 mediates the febrile response to localized inflammation in rats. J Physiol 525(3):653-661

Conn CA, Borer KT, Kluger MJ (1990) Body temperature rhythm and response to pyrogen in exercising and sedentary hamsters. Med Sci Sports Exerc 22(5):636-642 
Cooper KW, Brouwer S, Turnbull AV, Luheshi GN, Hopkins SJ, Kunkel SL, Rothwell NJ (1994) Tumor necrosis factor- $\alpha$ and fever after peripheral inflammation in the rat. Am J Physiol 267:R1431-R1436

Gordon CJ, Rowsey PJ (1998) Delayed febrile effects of chlorpyrifos: is there cross-tolerance to bacterial lipopolysaccharide? Toxicology 130(1):17-28

Gordon CJ (1994) Thermoregulation in laboratory mammals and humans exposed to antichlolinesterase agents. Neurotoxicol Teratol 16:427-453

Klir JJ, McClellan JL, Kluger MJ (1994) Interleukin-1 $\beta$ causes the increase in anterior hypothalamic interleukin-6 during LPS-induced fever in rats. Am J Physiol 266:R1845-R1848

Leon LR (2002) Molecular biology of thermoregulation (Invited Review): cytokine regulation of fever: studies using gene knockout mice. J Appl Physiol 92:2648-2655

Luheshi GN, Miller AJ, Brouwer S, Dascombe MJ, Rothwell NJ, Hopkins SJ (1996) Interleukin-1 receptor antagonist inhibits endotoxin fever and systemic interleukin-6 induction in the rat. Am J Physiol 270:E91-E95

Luheshi GN, Stefferl A, Turnbull AV, Dascombe MJ, Brouwer S, Hopkins SJ, Rothwell NJ (1997) Febrile response to tissue inflammation involves both peripheral and brain IL-1 and TNF- $\alpha$ in the rat. Am J Physiol 272:R862-R868

Michie HR, Manogue KR, Spriggs DR, Revhaug A, ODwyer S, Dinarello CA, Cerami A, Wolff SM, Wilmore DW (1988) Detection of circulating tumor necrosis factor after endotoxin administration. N Engl J Med 318(23):1481-1486

Nara M, Kanda T, Tsukui S, Inukai T, Shimomura Y, Inoue S, Kobayashi I (1999) Running exercise increases tumor necrosis factor-alpha secreting from mesenteric fat in insulin-resistant rats. Life Sci 65(3):237-244

Ostrowski K, Rhode T, Asp S, Schjerling P, Pedersen BK (1999) Pro- and anti-inflammatory cytokine balance in strenuous exercise in humans. J Physiol (Lond) 515:287-291
Ostrowski K, Rohde T, Zacho M, Asp S, Pedersen BK (1998) Evidence that IL-6 is produced in human skeletal muscle during prolonged running. J Physiol (Lond) 508:949-953

Pedersen BK (2000) Special feature for the Olympic: effects of exercise on the immune system: exercise and cytokines. Immunol Cell Biol 78:532-535

Pedersen BK, Toft AD (2000) Effects of exercise on lymphocytes and cytokines. Br J Sports Med 34:246-251

Rowsey PJ, Kluger MJ (1994) Corticotropin releasing factor is involved in exercise-induced elevation in core temperature. Psychoneuroendocrinology 19:179-187

Rowsey PJ, Borer KT, Kluger MJ (1993a) Role of prostaglandin in exercise-induced core temperature elevation in female SpragueDawley rats. Am J Physiol 265:R1121-R1125

Rowsey PJ, Borer KT, Kluger MJ (1993b) Tumor necrosis factor is not involved in the exercise-induced core temperature elevation in female Sprague-Dawley rats. Am J Physiol 265:R1351R1354

Rowsey PJ, Metzger BL, Carlson J, Gordon CJ (2003) Effects of exercise conditioning on thermoregulatory responses to repeated administration of chlorpyrifos. Environ Res 92:27-34

Rowsey PJ, Metzger BL, Gordon CJ (2001) Effects of exercise conditioning on thermoregulatory response to anticholinesterase insecticide toxicity. Biol Res Nurs 2(4):267-276

Satinoff E, Kent S, Hurd M (1991) Elevated body temperature in female rats after exercise. Med Sci Sports Exerc 23:1250-1253

Sprenger H, Jacobs C, Nain M et al (1992) Enhanced release of cytokines, interleukin-2 receptors and neopterin after long-distance running. Clin Immunol Immunopathol 63:188-195

Sugimoto N, Sakurada S, Shido O (2000) Changes in ambient temperature at the onset of thermoregulatory responses in exercise-trained rats. Int J Biometeorol 43:169-171

Yang Y, Gordon CJ (1996) Ambient temperature limits and stability of temperature regulation in telemetered male and female rats. J Therm Biol 21:353-363 